\title{
Promoting the immunization neighborhood: Benefits and challenges of pharmacies as additional locations for HPV vaccination
}

\author{
Mitchel Rothholz and Litjen (L. J.) Tan ${ }^{\mathrm{b}}$ \\ ${ }^{\mathrm{a} A m e r i c a n}$ Pharmacists Association, Washington, D.C., USA; ${ }^{\mathrm{b}}$ Immunization Action Coalition, St. Paul, MN, USA
}

ARTICLE HISTORY Received 9 November 2015; Revised 4 April 2016; Accepted 5 April 2016

KEYWORDS HPV; human papillomavirus; pharmacist immunization; public health; vaccination; vaccine preventable disease

The recently released CDC National Immunization Survey (NIS)-Teen 2014 data reveals that despite a slight increase in coverage in 2014 compared with 2013, human papilloma virus (HPV) vaccination rates remain low. ${ }^{1}$ Four out of 10 adolescent girls and 6 out of 10 adolescent boys haven't started the HPV vaccine series and therefore remain vulnerable to devastating cancers. While some state and local areas made significant increases in coverage, there was only a 3.3 percentage point overall increase nationally for first dose HPV vaccine coverage among adolescent girls. The adult immunization coverage rates for HPV vaccine don't look any better with only $36.9 \%$ of woman aged 19-26 in 2013 reporting receipt of $>1$ dose of HPV vaccine. For males, the numbers were much lower. In addition, completion of the 3 dose series by patients is low (adolescent females: $39.7 \%$, males: $21.6 \%$ ).

Improving HPV vaccination efforts focuses on 2 main strategies. ${ }^{2,3}$ The first is on improving the initiation of the 3 -dose vaccination series in adolescent and adult patients. The second is to ensure that those who have received the first dose of HPV vaccine continue to complete the full series of vaccination. Numerous strategies to improve the initiation of HPV vaccinations and to enhance completion of the 3-dose series, have been tried, with some strategies such as reducing missed opportunities through the introduction of decision support systems ${ }^{4}$ and utilizing patient reminder-recall systems, ${ }^{5}$ having a positive impact.

Additionally, some of other promising strategies include:

1. Establishing links between cancer organizations and immunization stakeholders;

2. Educating clinicians, including reminding them to take every opportunity to strongly recommend HPV vaccine, especially when they recommend meningococcal and Tdap vaccines;

3. Conducting public communication campaigns; and,

4. Instituting systems interventions, such as using registry or immunization information system (IIS)-based reminders for parents about upcoming shots.

What has become evident with these multiple efforts is that all strategies to improve HPV vaccination rates require access to vaccination providers who can give clear, consistent, and confident recommendations for vaccination.
Thus, collaborative efforts remain critical to promoting all recommended vaccinations for adolescent and adults, and in particular for improving HPV vaccination rates, as the completion of an HPV vaccination series requires more than just one visit to a provider. Indeed, opportunities exist for non-physician providers, like pharmacists, to play an important role in message delivery, education and administration of vaccine.

In 2013, the President's Cancer Panel issued its annual report titled "Accelerating HPV Vaccine Uptake:

Urgency for Action to Prevent Cancer." After documenting the case for HPV vaccination and the need for urgent action, the report's third section focused on accelerating HPV vaccine uptake in the United States. ${ }^{6}$ The President's Cancer Panel were unequivocal in their recommendations that improving HPV vaccination rates in the United States requires that the country maximizes access to HPV vaccination services, specifically mentioning the provision of the vaccine by providers, such as pharmacists, in coordination with the medical home. In particular, the report states that "Vaccines should be available where adolescents receive healthcare. It should be convenient to initiate and complete the HPV vaccine series, and cost should not be a barrier."

In particular, there is one aspect that must be addressed in order to open access to HPV vaccinations to the population. In 2015, there are 49 states and territories that authorize pharmacists to administer HPV vaccine. ${ }^{7}$ However, 30 of those have limitations based on age or other requirements, creating a barrier to ease of access. According to a recent annual immunization survey conducted by the American Pharmacists Association (APhA), 34\% of pharmacist respondents currently administer HPV vaccine, but of note, there is a strong desire among pharmacists to be engaged in HPV vaccination. ${ }^{8}$ There are more than 260,000 pharmacists in the US trained to administer immunizations across the lifespan and while not all are currently immunizing, they are well trained, available and ready to do their part in improving the public health of their communities. Thus it is important that the Panel's recommendation for states to enact laws and implement policies that allow pharmacists to administer vaccines to adolescents, including younger adolescents, be advanced. 
There has been significant national discussion of the importance of the "medical home," whether providing HPV vaccination in a pharmacy setting would jeopardize that "medical home," and this was indeed an item of discussion in the report from the President's Cancer Panel. There is no doubt of the importance of maintaining a medical home for the adolescent. However, as the Panel suggests, "providing additional venue choices may increase the likelihood that adolescents will receive all 3 HPV doses." For several years now, the APhA and the National Adult and Influenza Immunization Summit (www. izsummitpartners.org) have been promoting the term "immunization neighborhood" to describe a community approach to addressing immunization needs through collaboration, coordination, and communication among all immunization stakeholders. The goal is to meet the immunization needs of the patient and protect the public from vaccine-preventable diseases. ${ }^{9,10}$ Through this approach, an entire community can invest in assessing, recommending, administering, and/or referring patients to receive appropriate vaccines. It also supports the documentation of immunizations given, and the sharing and exchanging of immunization data among providers and can be focused on populations (pediatric, adolescent and adult) and/or preventable diseases (HPV, pertussis, etc.) to meet the needs of patients and the communities served. This concept is entirely consistent with the recent Standards for Adult Immunization Practice issued by the National Vaccine Advisory Committee. ${ }^{9}$ With respect to improving HPV vaccination coverage rates, factors such as patient education, enhanced patient comfort with the vaccine itself, access to trusted providers and to vaccination services have all been identified as important, and are all areas where immunizing pharmacists can make a positive impact. Thus, for example, by embracing the concepts of the immunization neighborhood, it may be possible to establish community relationships between physician and non-physician immunizers so that the different doses of the HPV vaccine can be delivered at locations that are more convenient for the patient.

It is difficult to provide confident vaccination recommendation if the patient's vaccination history is uncertain. To this end, it is essential that all providers ensure that vaccine administration information is entered into the state immunization information system (IIS) and/or the patient's medical record, if one exists, to ensure tracking toward completion of the HPV multi-dose series and reinforcement of vaccination messages.

Today's healthcare providers are being asked to see more patients and do more with fewer resources. The need for strategic collaborative relationships has never been greater. Patients benefit from the immunization neighborhood by gaining improved access, enhanced convenience, and targeted vaccination services for those at high risk. In the end, the immunization neighborhood concept benefits both providers and patients, and can make a difference in reducing preventable illnesses and ensuring healthier communities. HPV vaccination is a unique vaccine that cuts across pediatric, adolescent and adult populations and thus provides significant opportunity to identify, implement, and test strategies that will improve access to a multi-dose vaccination series across the lifespan within such an immunization neighborhood. Lessons learned as we implement the changes necessary to improve access to HPV vaccines will also serve to advise strategies to improve access to adult immunizations and to provide understanding of the necessary infrastructure to engage other providers to vaccinate children and adolescents in times of crisis.

In order to improve HPV vaccination rates, we need to have improved access to vaccination services for the adolescent patient coupled with a strong provider recommendation. The immunization neighborhood can accomplish this to improve HPV vaccination rates. By establishing the immunization neighborhood for each and every adolescent, we can provide access not only to both physician and non-physician immunization providers, but also access to educational opportunities to understand the tremendous safety and effectiveness of the HPV vaccines, and to emphasize that indeed, this is a cancer preventing vaccine. That said, all providers must recognize that their recommendation has tremendous impact on their patients. They must learn to develop a confident, clear and concise recommendation for HPV vaccination. This recommendation should be delivered along with any other adolescent vaccines that may be due at the visit, and no vaccine should be singled out for further attention. By harnessing our wealth of immunization providers and urging a strong recommendation from them, we can protect our nation against HPV-associated cancers and we should do so with urgency.

\section{Disclosure of potential conflicts of interest}

The authors reported no conflicts of interest.

\section{References}

[1] Reagan-Steiner S, Yankey D, Jeyarajah J, Elam-Evans LD, Singleton JA, Curtis CR, MacNeil J, Markowitz LE, Stokley S. National, Regional, State, and Selected Local Area Vaccination Coverage Among Adolescents Aged 13-17 Years-United States, 2014. MMWR Morb Mortal Wkly Rep 2015; 64:784-792; PMID:26225476; http:// dx.doi.org/10.15585/mmwr.mm6429a3

[2] Cook RL, Zhang J, Mullins J, Kauf T, Brumback B, Steingraber H, Mallison C. Factors associated with initiation and completion of human papillomavirus vaccine series among young women enrolled in Medicaid. J Adolesc Health 2010; 47:596-599; PMID:21094437; http://dx.doi.org/10.1016/j.jadohealth.2010.09.015

[3] Dorell CG, Yankey D, Santibanez TA, Markowitz LE. Human papillomavirus vaccination series initiation and completion, 2008-2009. Pediatrics 2011; 128:830-839; PMID:22007006; http://dx.doi.org/ 10.1542/peds.2011-0950

[4] Fiks AG, Grundmeier RW, Mayne S, Song L, Feemster K, Karavite D, Hughes CC, Massey J, Keren R, Bell LM, et al. Effectiveness of decision support for families, clinicians, or both on HPV vaccine receipt. Pediatrics 2013; 131:1114-1124; PMID:23650297; http://dx.doi.org/ 10.1542/peds.2012-3122

[5] Kharbanda EO, Stockwell MS, Fox HW, Andres R, Lara M, Rickert VI. Text message reminders to promote human papillomavirus vaccination. Vaccine 2011; 29:2537-2541; PMID:21300094; http://dx. doi.org/10.1016/j.vaccine.2011.01.065

[6] President's Cancer Panel. Accelerating HPV Vaccine Uptake: Urgency for Action to Prevent Cancer. President's Cancer Panel 2014. Available online at: http://deainfo.nci.nih.gov/advisory/ pcp/annualReports/HPV/PDF/PCP_Annual_Report_2012-2013.pdf Accessed November 7, 2015

[7] American Pharmacists Association. Pharmacist authority to immunize - by type of immunization. American Pharmacists Association 2015. Available online at: http://www.pharmacist.com/sites/default/files/files/ Pharmacist\%20authority\%20to\%20immunize\%20-\%20by\%20type\%20 of\%20immunization.pdf Accessed November 7, 2015

[8] Tanzi MG. Improving HPV vaccination rates: Real-world examples? American Pharmacists Association 2015. Available online at: http:// 
www.pharmacistsprovidecare.com/improving-hpv-vaccination-rates-r eal-world-examples Accessed November 7, 2015

[9] Recommendations from the National Vaccine Advisory Committee: Standards for Adult Immunization Practice. Public Health Rep 2014; 129:115-123; PMID:24587544
[10] Tanzi MG. It takes a village: NVAC standards emphasize importance of immunization neighborhood. Am Pharmacists Association 2014. Available online at: http://www.pharmacist.com/it-takes-vil lage-nvac-standards-emphasize-importance-immunization-neighbor hood Accessed November 7, 2015 